\title{
Performance Analysis of OFDM and OFDM-MIMO Systems under Fading Channels
}

\author{
Abdulbagi Elsanousi \\ Department of Electronics and Communication Engineering \\ Kocaeli University, Kocaeli, Turkey \\ alsnousi06037@hotmail.com
}

\author{
Sitki Ozturk \\ Department of Electronics and Communication Engineering \\ Kocaeli University, Kocaeli, Turkey \\ sitki59@gmail.com
}

\begin{abstract}
Fourth-generation (4G) wireless communication system is mainly based on MIMO (multiple input and multiple output) and OFDM (orthogonal frequency division multiplexing). The combination of these two techniques leads to a better system, known as OFDM-MIMO system, providing higher capacity and data rate. This paper demonstrates the BER (bit error rate) performance of OFDM and OFDM-MIMO system using convolution code to encrypt the data stream that can be sent over communication channels. Simulation is made on Matlab using three different channels (AWGN, Rician and Rayleigh). The simulation results show that the combined system has better performance compared to OFDM system. The practical part of this study was conducted by using two USRPs B210 (universal software radio peripheral) for transmitting and receiving, two antennas were used during transmission for both sides. The transmitted signal is successfully recovered.
\end{abstract}

Keywords-BER; OFDM; OFDM-MIMO; convolution code; Racian; AWGN; Rayleigh; USRP

\section{INTRODUCTION}

Wireless communication systems these days have to provide higher data rates to fulfill the high demand. MIMO is basically about improving the channel capacity by using multiple antennas. MIMO offers enhancement to data rate and channel capacity. MIMO-OFDM is an essential point of today's broadband wireless systems. In fact MIMO-OFDM technology has been being used in $4 \mathrm{G}, 4.5 \mathrm{G}$ and is going to be used in 5G. Current generation wireless systems, such as 4G, support data rates up to $1 \mathrm{Gbps}$, which seems to be insufficient for high demand. In order to achieve this requested data rate, the near future generation 5G plans to use MIMO for signal processing and OFDM for radio technologies [1]. Upcoming mobile and wireless applications will need significantly higher data rates and cut-price cost per transmitted bit compared to $3 \mathrm{G}$ systems. Systems using single antenna fail to meet the requirements on data rate, link quality, spectral efficiency etc. In MIMO technology high gain is ensured for both channel capacity and reliability. The use of multiple antennas instead of a single antenna allows to enhance coverage, data transfer rate, security of the radio networks [2]. The BER of convolutional coded orthogonal frequency-division multiplexing system is analyzed in [3]. The BER is evaluated for different modulation schemes. This paper gives a generalized BER analysis of OFDM and MIMO-OFDM systems.

\section{PRINCIPLES OF OFDM SYSTEM}

OFDM is a way of encoding digital data on multiple carrier frequency and is essential to current generation wireless technology [4]. OFDM is a multicarrier transmission technique based on the theory of frequency division multiplexing. It was introduced for $3 \mathrm{G}$ networks to improve overall link capacity but later on it became a part of the current generation of wireless technology [5]. In this technique spaced subcarriers are used to carry data on several parallel data streams or channels, and that is why it is called a multicarrier frequency technique. This helps in minimizing the signal fading as much as possible and provides enhanced data rates [6]. The main idea behind OFDM is to divide carriers to subcarriers that are transmitted in parallel. In the OFDM system [7], the data are sent to the serial to parallel block where signal converts to a set of data blocks. Then this set of parallel data blocks are sent to constellation mapper which can be of three or four types (BPSK, QPSK, QAM) and then this data need to be modulated [4]. Once it is modulated it is passed on through the IFFT block whose prior and most important work is to orthogonalize the data [9]. It takes a number of symbols that are the total number of subcarriers which are basically multicarrier, and provides orthogonal sinusoidal. Each of these outputs has different frequencies like a multi carrier frequency technique which has different orthogonal frequency onto it and once it comes out of IFFT, it goes onto the parallel to serial conversion block, where it can get one total serial signal [10]. Once it is ready to be sent to the channel, the channel sends it to the receiver, which receives it and the inverse process happens first by deleting the cyclic prefix and then reversing the IFFT time domain back into the frequency domain [3]. Once we get the frequency domain parallel signals we equalize them and send them back to the constellation demapper, where the entire demodulation work occurs. After demodulating the entire signal and getting the parallel data, the signal converts finally to serial to get the final data [11].

\section{MIMO WIRELESS TECHNOLOGY OVERVIEW}

An antenna system, called single input single output (SISO), which is a transmitting and receiving antenna, is usually also known as conventional mobile radio communication system. Greater network capacity, higher data rate and better quality are needed in modern telecommunication 
systems. Both bandwidth and transmit power need to be increased, in order to improve the capacity of the SISO system. MIMO system is well known as a technique to increase the capacity without changing the transmitted power and original bandwidth requirements [12]. The basic idea is to improve channel capacity by using multiple antennas. MIMO gives significant enhancement to data rate and channel capacity. MIMO communication promises large gains for both channel capacity and reliability. Using multiple antenna instead of a single one can enhance data transfer rate, coverage, security and overall performance of radio networks. MIMO technology uses two main techniques known as spatial multiplexing and spatial diversity. In the spatial diversity form of MIMO, the same data is sent over different transmitters and this leads to augmented reliability. While in the spatial multiplexing the bites stream to be transmitted are divided or demultiplexed into several data segments. These segments are then transmitted through different antennas simultaneously and this leads to increased data rate [13].

\section{MultipAth FADING}

Unlike a wired channel, which uses a fixed path, the signals in a wireless channel can reach a user using multiple paths. All these signals known as multipath components may have different channel gains and time delay. This combined effect causes what we know as multipath fading. Fading, usually called small scale fading, is caused by multipath signal, when signal transmits from source to destination. This signal may be constructive or destructive [6]. The time delay between first signal at the receiver always (line of sight) and the last signal received from multipath is called delay spread [14].

\section{A. Rayleigh Model}

The common distribution that is used to describe statistical time varying nature of received envelope of flat fading signal is the Rayleigh distribution. The main idea of the Rayleigh flat fading channel model is that there is no line of sight between transmitter and receiver [15]. The received signal is reflected or scattered by multipath. The probability density function of Rayleigh distribution is given by:

$$
P(x)=\left\{\begin{array}{c}
\frac{x}{\sigma^{2}} \exp \left(-\frac{x^{2}}{2 \sigma^{2}}\right) 0 \leq x \geq \infty \\
0 x<0
\end{array}\right.
$$

where $\sigma$ and $\sigma^{2}$ are received root-mean-square and a.c power of the received signal respectively [16]. The Rayleigh channel coefficients computed according to (2).

$$
h=\left(\frac{1}{\sqrt{2}}\right) *(N(0,1)+j N(0,1))
$$

\section{B. Rician Distribution}

The Rician fading occurs when there is a dominant non fading signal component like a line of sight propagation path. When the power in the dominant component decreases to zero, Rician distribution can be seen as Rayleigh distribution. The ratio between the line of sight and the other diffused components is known as Rice factor, denoted by $\mathrm{KR}$ and expressed as follows $K_{R}=A^{2} /\left(2 \sigma^{2}\right)$ [15].

$$
P(x)=\left\{\begin{array}{c}
\frac{x}{\sigma^{2}} \exp \left(-\frac{x^{2}+A^{2}}{2 \sigma^{2}}\right) I_{0}\left(\frac{x A}{\sigma^{2}}\right) A \geq 0, x \geq 0 \\
0 x<0
\end{array}\right.
$$

where $A$ represents the peak amplitude of the dominant component while and $I_{0}($.$) is the modified Bessel function of$ the first kind and zero order [12]. The Rician channel coefficients can be computed according to (4), where $K_{0}<1$.

$h=K_{0}+\sqrt{\frac{1-K_{0}^{2}}{2}}(N(0,1)+j \cdot N(0,1))$

\section{The Need for Equalization}

Equalization is used for compensating inter symbol interference. Also, an equalizer within receiver compensates for the average range of expected channel amplitude and delay characteristic [17]. Equalizers must be adaptive as the channel is generally unknown and time varying. In the current study we used minimum mean-squared error (MNSE). Basically the MNSE equalizer for each subcarrier is defined as

$$
\hat{S}=\frac{r(k) \cdot H(k)^{*}}{E_{0}+\left(H(k)^{*} \cdot H(k)\right)}
$$

where $E_{0}$ is noise power, $r(k)$ is the received symbol, $H(k)$ is the channel in frequency domain at subcarrier $k$.

\section{OFDM-MIMO SYSTEM}

In the future broadband wireless techniques will provide big data rates and will improve channel performance. These channels may be time or frequency selective. We know that MIMO system has the capability to enhance bandwidth and diversity. Adding OFDM can help us decrease the effects due to multipath fading. The use of MIMO OFDM is to send data over wireless communications and to overcome frequency selective channel effect [18]. The main idea behind the use of OFDM on each subcarrier is to deal with narrowband fading by transforming frequency-selective fading channels into flat channels. MIMO algorithms can be implemented in broadband transmission with the help of the combined MIMO and OFDM techniques [19]. At the transmitter, OFDM modulated data can be transmitted over multiple antennas simultaneously using a MIMO OFDM system. At the receiver, OFDM demodulation is required then recovery of the signal is performed by decoding techniques on each of the sub-channels originated from all the transmitting antennas [18]. Using MIMO OFDM, suppliers will have the opportunity to apply a broadband wireless access (BWA) system which possesses non-line-of-sight (NLOS) features. Other advantages of MIMO-OFDM are that it provides flexibility and big throughput, and also, in multiuser application, it facilitates a big number of users to access the base station or access point. By using OFDM modulator the signal is modulated, and then transmitted by the MIMO system. The inverse process can be used to recover signals [12]:

$$
\bar{y}=H \cdot \bar{x}+\bar{n}
$$

where $\bar{y}$ and $\bar{x}$ are received and transmitted vectors respectively, while $H$ and $\bar{n}$ are channel matrix and noise.

\section{UNIVERSAL SOFTWARE RADIO PERIPHERAL}

Software-defined radio is a technology that enables users to experiment with radio waves. The USRP B210 is a fully 
integrated single board software-defined radio that connects to a host pc through a USB 3.0 interface. This entire USB powered device provides continuous coverage from $70 \mathrm{MHz}$ to $6 \mathrm{GHz}$ setting a new standard for wideband access. The USB 3.0 interface can stream up to $56 \mathrm{MHz}$ of instantaneous bandwidth. The b200 is capable full duplex communication and the b210 supports $2 \times 2$ MIMO [20]. Figure 1 shows USRP transmitter and receiver connected with two laptops.

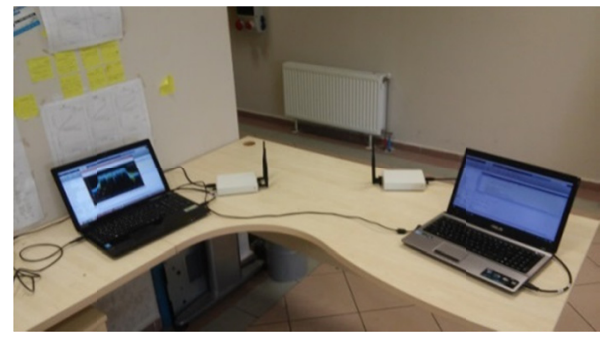

Fig. 1. USRP transmitter and receiver

\section{A. Transmit Path}

The host computer builds and sends baseband I/Q signals to the device through either USB 3.0 or 2.0 connection and then the digital up converter (DUC) mixes, filters, and interpolates the signal to $61.44 \mathrm{MS} / \mathrm{s}$. Then the digital-to-analog converter (DAC) converts the incoming signal to analog. The main goal of the band pass filter is to decrease the noise and high frequency components in the signal. The purpose of the mixer up is converting the signals to a user-specified RF frequency. The phase-locked loop (PLL) controls the voltage-controlled oscillator (VCO) so that the device clocks and local oscillator (LO) can be frequency-locked to a reference signal. At the last step the amplifier amplifies the incoming signal and sends it over the antenna.

\section{B. Receive Path}

The incoming signal is amplified by both low-noise amplifier and drive amplifier. The phase-locked loop controls the VCO so that the device clocks and LO can be frequencylocked to a reference signal. The idea from the mixer down is converting the signals to the baseband in-phase (I) and quadrature-phase (Q) components. After that the band pass filter decreases noise and high frequency components in the signal. Then the signal enters the analog-to-digital converter (ADC) which responsible to digitize the I and $\mathrm{Q}$ data. The digital down converter (DDC) mixes, filters, and decimates the signal to a user-specified rate. The down converted samples are moved to the host computer through USB connection which is USB 3.0 or USB 2.0 connection. This study is done by using two USRPs for transmitting and receiving with two antennas for each USRP. The transmitted signal is successfully recovered. The experiment is set to measure the gain and frequency. Algorithm 1 illustrates the scenario of the code.

- Algorithm 1: Psuedo-code of the OFDM-MIMO with USRP B210

$$
\begin{aligned}
& \text { Begin } \\
& 1 \quad \text { randi }(x) \leftarrow \text { Generate bits } \\
& 2 \quad \text { convenc }(x) \leftarrow \text { convolutional encoding }
\end{aligned}
$$

3 reshape $(\mathrm{x}) \leftarrow$ Symbols construction

4 bi2de(x)

5 reshape $(\mathrm{x}) \leftarrow$ Serial to parallel

$6 \quad \operatorname{sort}(\operatorname{rand}(\mathrm{x})) \leftarrow$ Interleaver

$7 \quad$ qammod $(\mathrm{x}) \leftarrow$ QAM Modulation

8 if $(\mathrm{fft}(\mathrm{x})) \leftarrow$ OFDM Modulation

$9 \quad \mathrm{x}($ cyclic prefix $) \leftarrow$ Adding cyclic prefix

$10 \mathrm{x} \leftarrow$ Parallel to serial

$11 \quad(1 /$ sqrt $\{2\}) * \mathrm{~N}(0,1)+\mathrm{jN}(0,1) \leftarrow$ Channel

12 reshape $(\mathrm{x}) \leftarrow$ Serial to parallel

$13 \mathrm{x}$ (cyclic prefix) $\leftarrow$ Remove cyclic prefix

$14 \mathrm{fft}(\mathrm{x}) \leftarrow$ OFDM Demodulation

$15 \mathrm{r}(\mathrm{k}) \operatorname{conj}(\mathrm{H}(\mathrm{k}) /(\mathrm{n}, \mathrm{p} \mathrm{H}(\mathrm{k}) \operatorname{conj}(\mathrm{H}(\mathrm{k})) \leftarrow$ equalizer

16 qamdemod( $\mathrm{x}) \leftarrow$ QAM Demodulation

17 find $(\mathrm{x}) \leftarrow$ deinterleaver

18 de2bi $(x) \leftarrow$ Parallel to serial

$19 \operatorname{vitdec}(\mathrm{x}) \leftarrow$ Convolution Decoder

TABLE I. SIMULATION PARAMETERS OF OFDM SYSTEM

\begin{tabular}{|c|c|}
\hline Parameter & Value \\
\hline Subcarriers & 64 \\
\hline Digital Modulation & QAM modulation \\
\hline Signal to noise ratio & $(0-30) \mathrm{dB}$ \\
\hline Size of frame & 256 \\
\hline Number of frames & 100 \\
\hline Number of pilots & 4 \\
\hline Cyclic prefix & $(16) 25 \%$ \\
\hline Channel fading & Rician, Rayleigh and AWGN \\
\hline
\end{tabular}

\section{EXPERIMENTAL RESULTS}

Bit error rate of OFDM system using convolution code and without convolution code in three different channels is compared. Figure 2 shows the BER of OFDM with and without $\mathrm{CC}$ in AWGN channel. BER with $\mathrm{CC}$ significantly outperforms the BER without CC for 2QAM, 16QAM and 64QAM.

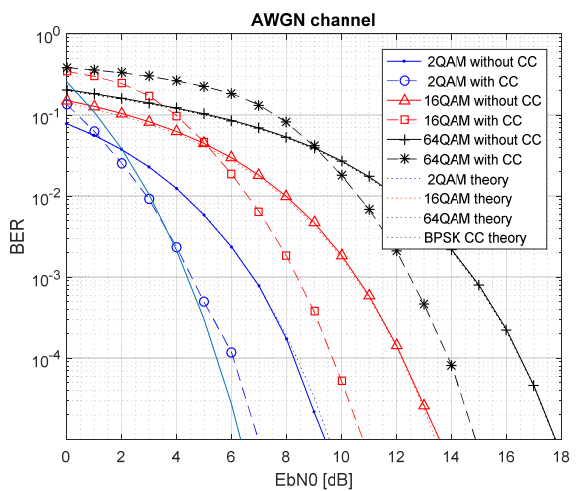

Fig. 2. BER vs. Signal to Noise Ratio (SNR) for OFDM system with and without convolutional code

Figure 3 illustrates BER with SNR in Rayleigh channel for the same modulation. The two figures reveal that BER of the OFDM system in AWGN channel outperforms Rayleigh channel. Figure 4 shows BER of OFDM with CC and without CC for QPSK, 16QAM and 64QAM in Rician channel. QPSK has good performance compare with other modulations. 


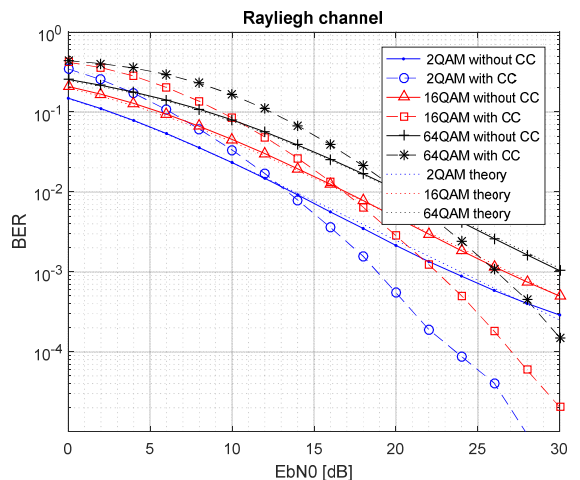

Fig. 3. BER vs. SNR for OFDM system with and without convolutional code

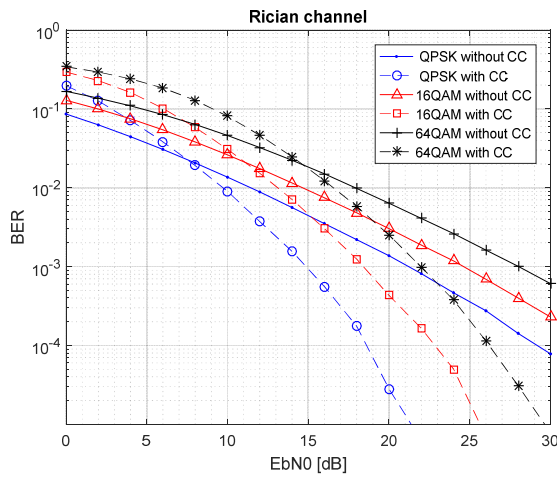

Fig. 4. BER vs. SNR for OFDM system with and without convolutional code

Figures 5-7 show BER of OFDM-MIMO system with SNR in the same three different channels with convolutional code and without convolutional code. Overall, results show that BER decreases when the number of antennas increases. This is because MIMO technology uses spatial multiplexing and spatial diversity. In case of spatial diversity the same data is sent over different transmitters and this leads to augmented reliability, while in the spatial multiplexing the bite stream to be transmitted is divided or de multiplexed into several data segments. These segments are then transmitted through different antennas simultaneously, and this leads to increased data rate. One of the drawbacks of this system is that the use of more antennas can lead to more power consumption.

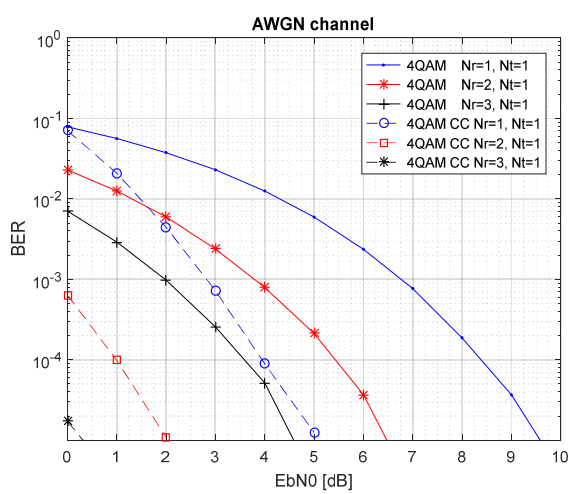

Fig. 5. BER vs. SNR for OFDM-MIMO system with convolutional code

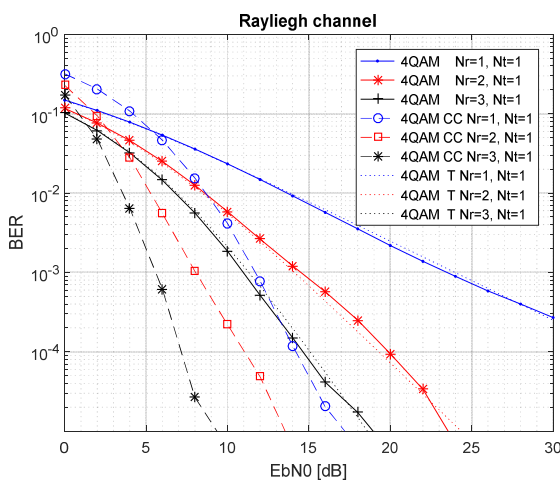

Fig. 6. BER vs. SNR for OFDM-MIMO system with convolutional code

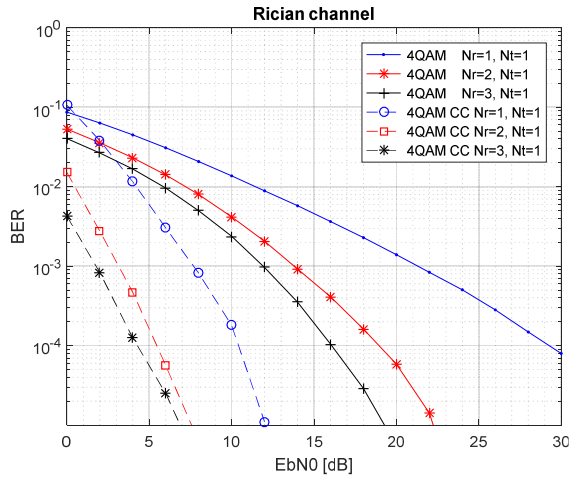

Fig. 7. BER vs. SNR for OFDM-MIMO system with convolutional code

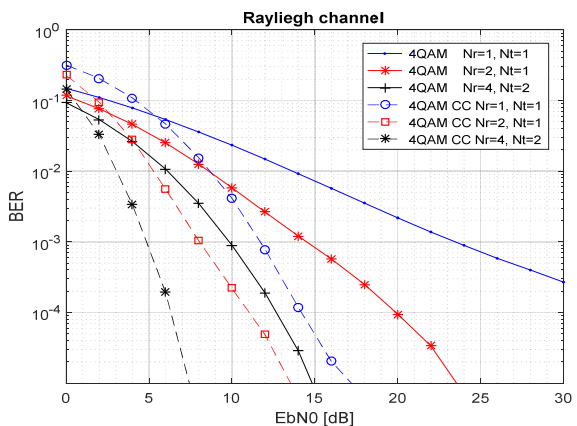

Fig. 8. BER vs. SNR for OFDM-MIMO system with convolutional code

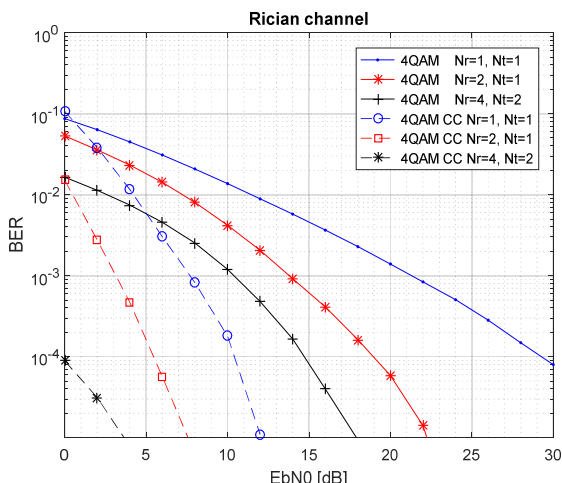

Fig. 9. BER vs. SNR for OFDM-MIMO system with convolutional code 
Figure 10 illustrates the power spectrum of the two-channel LTE signal. During transmission two antennas are used for both sides. MIMO 2x2 is used to offer spatial multiplexing and diversity. In the simulation, diversity technique is used to offer high reliability. In order to recover the transmitted signal correctly parameters such as gain, frequency and position of the antennas should be considered. Spectrum analysis of the received signal is shown to be equivalent to the one that is transmitted and shown in Figure 11. Physical downlink control channel (PDCCH) demodulation of the QPSK received signal is shown in the same figure.

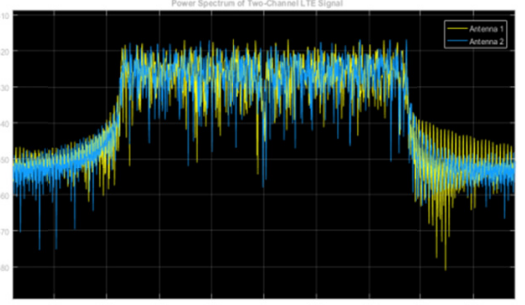

Fig. 10. Power spectrum of the two-channel LTE signal

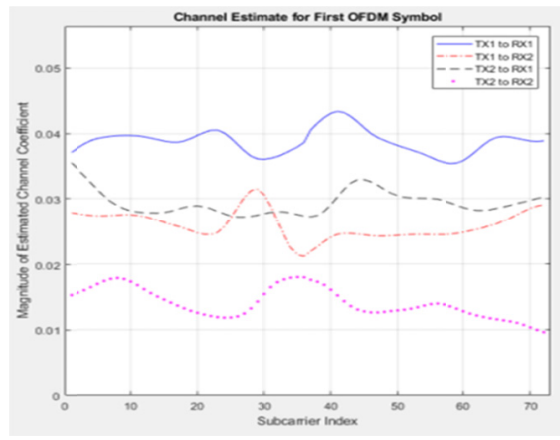

(a)
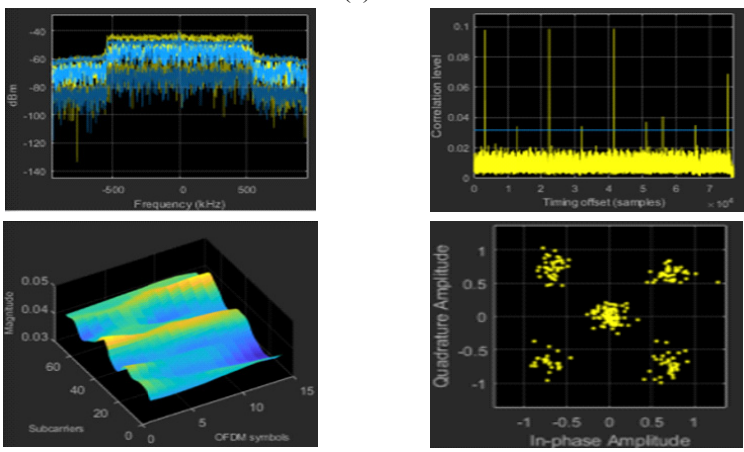

(b)

Fig. 11. Received signal spectrum

\section{CONCLUSION}

BER performance of both OFDM and OFDM-MIMO systems using convolutional channel coding and different digital modulation schemes has been shown in this study. Time-invariant frequency selective channel, the way of coding data, Viterbi, trace-back-length, hard or soft decision decoding, code-rate, interleaver and type of equalizer were considered as parameters during the simulation. The lowest BER can be obtained at the highest number of transmitting and receiving antennas. The channel order increases due to the increase of multi path channel fading which increases BER as well. The results show that OFDM-MIMO system has better performance than OFDM system. Regarding channels, Racian shows better performance than Rayleigh while AWGN gives the best. This work has been conducted using two USRPs for transmitting and receiving with two antennas each. The transmitted signal is successfully recovered.

\section{REFERENCEES}

[1] N. Sethy, S. Swain, "BER analysis of MIMO-OFDM system in different fading channel", International Journal of Application or Innovation in Engineering \& Management, Vol. 2, No. 4, pp. 405-409, 2013

[2] S. K. Borra, S. K. Chaparala, "Performance Evalution of OFDM System with Rayleigh, Rician and AWGN channels", International Journal of Innovative Research in Computer and Communication Engineering, Vol. 5, No. 7, pp. 13222-13227, 2017

[3] N. H. Maruf Bhuyan, M. Ahmed, M. Faisal, M. Salauddin, "Bit Error Rate Performance Analysis of Convolutional Coded Orthogonal Frequency-Division Multiplexing System", 57th International Scientific Conference on Power and Electrical Engineering of Riga Technical University, Riga, Latvia, October 13-14, 2016

[4] H. G. Ryu, T. P. Hoa, N. T. Hieu, J. Jianxue, "BER Analysis of Clipping Process in the Forward Link of the OFDM-FDMA Communication System", IEEE Transactions on Consumer Electronics, Vol. 50, No. 4 , pp. 1058-1064, 2004

[5] R. P. Kumar, "Improving the Bit Error Rate of OFDM using Convolutional codes", International Journal of Advanced Research Foundation, Vol. 1, No. 2, pp. 4-8, 2014

[6] G. B. Umesha, M. N. Shanmukha Swamy, "Performance of OFDM System for Wireless Communication through Channel Estimation", International Journal of Electronics, Electrical and Computational System, Vol. 6, No. 1, pp. 21-26, 2017

[7] N. H. Maruf Bhuyan, J. Uddin, M. Hoque, M. A. Habib, "Performance Analysis of Wi-MAX:Modulation Scheme Versus Bit Error Rate", International Journal of Electronics \& Communication Technology, Vol. 3, No. 4, pp. 188-191, 2012

[8] J. Korhonen, Introduction to $3 \mathrm{G}$ Mobile Communications, 2nd ed, Artech House, 2003

[9] S. Gravano, Introduction to Error Control Codes, Oxford University Press, 2001

[10] S. Singh, S. Singh Rathore, "Simulation and Analysis of ConvolutionCoded OFDM under AWGN Noisy Channel", International Journal of Engineering Research \& Technology, Vol. 3, No. 8, pp. 1489-1493, 2014

[11] T. F. Wong, T. M. Lok, Theory of Digital Communication, University of Florida, 2004

[12] Y. I. Hammadi, Study And Design OF MIMO-OFDM System Operating Over Wireless Channel, MSc Thesis, Cankaya University, 2013

[13] J. A. Shokouh, Receives of Antenna Selection for NoiseLimited/Interference MIMO Channels, $\mathrm{PhD}$ Thesis, University of Waterloo, 2008

[14] A. Goldsmith, Wireless Communications, Cambridge University Press, 2005

[15] S. Gaur, Analysis of Advanced Diversity Receivers for Fading Channels, MSc Thesis, Virginia Polytechnic Institute and State University, 2003

[16] P. Podder, T. Zaman Khan, M. Haque Khan, M. Muktadir Rahman, "BER Performance Analysis of ofdm-bpsk, qpsk, qam Over Rayleigh Fading Channel \& Awgn Channel," International Journal of Industrial Electronics and Electrical Engineering, Vol. 2, No. 7, pp. 1-5, 2017

[17] D. Neumann, T. Wiese, W. Utschick, "Learning the MMSE Channel Estimator", IEEE Transactions on Signal Processing, Vol. 66, No. 11, pp. 2905-2917, 2018 
[18] H. Sampath, S. Talwar, J. Tellado, V. Erceg, A. Paulraj, "A fourthgeneration MIMO-OFDM broadband wireless system: design, performance, and field trial results", IEEE Communications Magazine, Vol. 40, No. 9, pp. 143-149, 2002

[19] J. Jayakumari, "MIMO-OFDM for 4G Wireless Systems", International Journal of Engineering Science and Technology, Vol. 2, No. 7, pp. 2886-2889, 2010

[20] National Instruments, USRP-2901 Block Diagram, available at: http://www.ni.com/documentation/en/software-defined-radio-device/ latest/usrp-2901/block-diagram/ 